\title{
Deciphering Nonenzymatic RNA Polymerization through Crystallography
}

\author{
Wen Zhanga,b, Jack W. Szostak a,b,c,,
}

aHoward Hughes Medical Institute, Department of Molecular Biology and Center for Computational and Integrative Biology, Massachusetts General Hospital, 185 Cambridge Street, Boston, Massachusetts 02114, United States

bepartment of Genetics, Harvard Medical School, 77 Avenue Louis Pasteur, Boston, Massachusetts 02115, United States

'Department of Chemistry and Chemical Biology, Harvard University, 12 Oxford St., Cambridge, Massachusetts 02138 , United States

Many high resolution crystal structures have contributed to our understanding of the reaction pathway for catalysis by DNA and RNA polymerases, but the structural basis of nonenzymatic template-directed RNA replication has not been studied in comparable detail. Here we present crystallographic studies of the binding of ribonucleotide monomers to RNA primer-template complexes, with the goal of improving our understanding of the mechanism of nonenzymatic RNA copying. Using our synthetic unreactive phosphonate-linked analog of activated monomer, we obtained the structures of RNA primer-template complexes with the monomers bound. Our structures demonstrate the versatile binding motifs of activated mononucleotide substrate in nonenzymatic RNA polymerization, which could significantly influence the rate and fidelity of RNA replication, and they also illustrate the structural rationale of the great catalytic function of the downstream helpers. In addition, our time-resolved structures successfully integrate several static RNA-substrate structures into a molecular "movie" following the reaction process, and it clearly reveals the mechanism of RNA nonenzymatic replication. Against the traditional opinion that the catalysis of downstream activation is based on the noncovalent leaving group-leaving group effect, our time-resolved structures demonstrate that the facilitation is from the formation of imidazoliumbridged intermediate. Our structures provide the powerful tool and fundamental evidence for the RNA self-replication mechanistic studies. 